Material Behaviour

\title{
Biodegradability Study of Polylactic Acid/ Thermoplastic Polyurethane Blends
}

Vladislav Jašo ${ }^{\mathrm{a}}$, Gregory Glenn ${ }^{\mathrm{b}}$, Artur Klamczynski ${ }^{\mathrm{b}}$, Zoran S. Petrovića

${ }^{\mathrm{a}}$ Kansas Polymer Research Center, Pittsburg State University, Pittsburg, KS 66762, USA

bUSDA, ARS, PWA, WRRC-BCE, Albany, CA 94710, USA

Correspondence to:

Vladislav Jašo

Kansas Polymer Research Center

Pittsburg State University

1701 S. Broadway

Pittsburg, KS 66762

Phone: +1 620-235-6591

Fax: +1 620-235-4049

E-mail: vjaso@pittstate.edu 


\section{ABSTRACT}

Blends with varied ratio of polylactic acid (PLA) and thermoplastic polyurethane (TPU) were prepared by melt blending. The PLA content in blends was 20, 40, 60 and 80 wt\%. Samples of pure PLA and TPU that underwent the same thermal treatment were also prepared. Biodegradation was examined by respirometry. Pure TPU started to degrade immediately due to degradation of the low molecular weight plasticizer in the polymer. Pure PLA, on the other hand, exhibited an incubation period after which degradation progressed rapidly and was almost complete after 70 days. The degradation profile of the blends can be correlated to their morphology. Samples with a co-continuous morphology initially degrade at a higher rate than the rest of the samples due to the higher exposure of the TPU phase in these blends.

Key words: polylactic acid; thermoplastic polyurethane; biodegradability; morphology.

\section{INTRODUCTION}

Biodegradability is an important material property, especially as it relates to environmental concerns. Polylactic acid (PLA) is a known biodegradable polymer that has found wide application in many areas. It is also biocompatible which makes it ideal for use in the biomedical field ${ }^{1}$. Much broader use of PLA, however, is limited by its brittleness. This can be overcome by blending the PLA with an elastomer such as thermoplastic polyester-urethane. Thermoplastic polyurethanes (TPU) have found applications in the production of medical devices because of their hemocompatibility and excellent mechanical properties ${ }^{2-6}$. These two materials (PLA, TPU) have the potential to be compatible and miscible to some degree as they 
both have ester bonds in their main chain. Furthermore, the carbamate from the TPU hard segment can form hydrogen bonds with PLA. These two materials could complement each other very well in blends since PLA has high tensile strength and hardness while TPU has high elongation and toughness.

In our previous work ${ }^{7}$ we obtained two classes of materials by blending PLA with an elastomer. At low concentrations of the PLA, a tough thermoplastic material was formed and at high concentrations of PLA, a strong, rigid material was made. Studies of the blend morphology revealed that globular PLA dispersed in a TPU matrix is formed at low concentrations of PLA (10-30 wt\%). At concentrations of 40 wt\% and 50 wt\% of PLA, a co-continuous morphology was exhibited, i.e., the TPU and PLA phases were intermingled and stretched from one end of the sample to the other. At higher concentrations of PLA (60-80 wt\%) a globular morphology was once again observed but with TPU globules dispersed in a PLA matrix. The blend morphology was found to exert a key influence on the dynamic-mechanical and mechanical properties of the blends. Blend morphology would also be expected to affect the degradation of the blends. The rate of biodegradation of a blend is dependent on experimental conditions such as temperature, $\mathrm{pH}$, surface area of the sample, water absorption and the chemical properties. For instance, polymers like PLA first absorb water and then hydrolytic cleavage of ester bonds occurs, generating chain fragments with acidic end groups. The result of this process is a decrease in molecular weight of the PLA and an accelerated rate of biodegradation.

The objective of this work was to study the influence of composition and morphology of polylactic acid/ thermoplastic polyurethane blends on the rate of biodegradation. 


\section{MATERIALS}

Elastollan C 60A 10WH (BASF Polyurethanes $\mathrm{GmbH}$, Lemförde, Germany) is a plasticized thermoplastic polyester urethane having nominal Shore A hardness of 64 and density of 1.17 $\mathrm{g} / \mathrm{cm}^{3}$. The plasticizer content is around $30 \mathrm{wt} \%$. The structure of the plasticizer was not revealed by the manufacturer but our extraction studies indicated it was a phthalate-type product, probably dibutyl phthalate.

The PLA used in this study(6201D, Nature Works, Minnetonka, MN, USA) was a fibergrade PLA with a density $1.24 \mathrm{~g} / \mathrm{cm}^{3}$ and melt flow index of $15-30 \mathrm{~g} / 10 \mathrm{~min}$ (at load $2.16 \mathrm{~kg}$ ).

\section{EXPERIMENTAL}

The preparation of the blends and their morphology were described earlier ${ }^{7}$. Respirometry was performed as per ASTM D5338. In short, compost was purchased locally and sieved (20 mesh) before use. The compost was adjusted to $60 \%(w / w)$ moisture and added to each individual reaction chamber $(500 \mathrm{~mL})$. Polymer samples were milled to a fine consistency (ca. $100 \mu \mathrm{m}$ ) using a Wiley mill. Milled sample $(0.2 \mathrm{~g})$ was added to the compost in each reaction chamber and thoroughly mixed. Sample chambers were connected to an automated and fully computerized closed-circuit Micro-Oxymax Respirometer System (Columbus Instruments, Columbus, $\mathrm{OH}$ ). Temperature of the samples was maintained by placing the 500 $\mathrm{mL}$ reaction chambers in a temperature-controlled bath of propylene glycol. This system was used to monitor the concentration of $\mathrm{CO}_{2}$ gas generated within each reaction chamber over time. Experiments were carried out at an elevated temperature $\left(58^{\circ} \mathrm{C} \pm 0.2^{\circ} \mathrm{C}\right)$ over a period of 70 days. Control samples were run in triplicate to measure the background $\mathrm{CO}_{2}$. The 
background $\mathrm{CO}_{2}$ levels were subtracted from the sample data. Each sample was run in duplicate and averaged.

\section{RESULTS AND DISCUSSION}

The degradation of the blends was foremost dependent on their composition and morphology. Results of biodegradation are shown in Figure 1. Two groups of materials can be defined by their degree of degradation: high degradation (weight loss above $50 \%$ after 70 days) is shown by samples with 50, 60 and $100 \%$ PLA, while all other displayed slower degradation (weight loss $30 \%$ or lower). Biodegradation of pure PLA exhibits an induction period of around 20 days, after which it proceeds very rapidly. It degrades almost $100 \%$ after 70 days. Pure TPU on the other hand, starts to degrade immediately, probably as a result of the fast degradation of the low molecular plasticizer. Final weight loss during degradation of TPU was around $26 \%$ after 70 days. The loss coincides with the amount of plasticizer present, but it is not clear how much the polyurethane contributed to it. In our previous study ${ }^{8}$ of another type of polyester, urethane total weight loss was about $5 \%$ after 60 days. However, the current polyurethane is much softer with higher ester to urethane bond ratio, and thus more biodegradable. Biodegradation of phthalates by bacteria and fungi under aerobic, anoxi, and anaerobic conditions has been confirmed by numerous studies ${ }^{9}$.

Figure 1. Mineralization of pure PLA, TPU and their blends.

Blends with co-continuous morphology (PLA 40 and PLA 50) show higher initial degradation rate than other blends (Figure 2). The reason for this is higher exposure of the two phases to the environment. High exposure of the TPU phase meant that degradation could start 
almost immediately by degradation of the plasticizer. The relatively high PLA content and its exposure to degradation because of the co-continuous morphology enabled degradation of these samples to continue at high rate during the whole period of 70 days (Figure 3).

Figure 2. Mineralization of PLA, TPU and their blends during first 20 days.

Figure 3. Rate of biodegradation of PLA, TPU and their blends.

The sample with $40 \mathrm{wt} \%$ of PLA had a high content of TPU so its initial degradation rate was higher than at later stages that were dominated by the degradation of PLA. For the same reason, the sample with $50 \mathrm{wt} \%$ of PLA had a higher degradation rate at later stages than at the initial stage. On the other hand, the sample with 60 wt\% of PLA which had TPU globules dispersed in the PLA matrix had a much lower initial degradation rate because of the protective layer of PLA. The rate of degradation was much higher at later stages (Figure 3).The sample with 80 wt\% of PLA displayed a lag in degradation, much like the pure PLA sample, since embedded TPU globules were not accessible for early degradation. The sample with $20 \mathrm{wt} \%$ of PLA started to degrade immediately but at a much lower rate than pure TPU (Figure 2) due to the presence of PLA. Once the degradation of $20 \mathrm{wt} \%$ PLA sample started, it reached only 20 wt\% weight loss but with a tendency to continue the process further (Figure 1). The maximum degradation rate was displayed by all samples after 20-40 days, shifting to longer times with increasing urethane content.

\section{CONCLUSIONS}

Respirometry was employed to study biodegradation of blends with different PLA/TPU ratios and different morphologies. Biodegradation of pure PLA starts after 20 days of incubation 
time and proceeds very rapidly, degrading almost completely after 70 days. Polyester urethane, on the other hand, starts to degrade instantly but slows down and ultimately does not display more than $30 \%$ mineralization after 70 days. It was found that morphology has a strong influence on degradation rate of PLA/TPU blends. Blends with co-continuous morphology degraded at higher initial rate than other blends that had globular morphology. The PLA content in the blend influences its ultimate level of degradation.

\section{ACKNOWLEDGMENT}

This work was supported by a grant from the United States Department of Agriculture (USDACSREES 2010-38924-20706).

\section{REFERENCES AND NOTES}

1. X. Zhao, L. Ye, P. Coates, F. Caton-Rose, M. Martyn. Structure and blood compatibility of highly oriented poly(lactic acid)/thermoplastic polyurethane blends produced by solid hot stretching. Polym Adv Technol 2013; 24 (9):853.

2. Q. Cao, Y. Cai, B. Jing, P. Liu. Structure and Mechanical Properties of Thermoplastic Polyurethane, Based on Hyperbranched Polyesters. J Appl Polym Sci 2006; 102 (6): 5266.

3. A. Simmons, J. Hyvarinen, L. Poole-Warren. The effect of sterilisation on a poly(dimethylsiloxane)/poly(hexamethylene oxide) mixed macrodiol-based polyurethane elastomer. Biomaterials 2006; 27 (25): 4484. 
4. S. Gogolewski, K. Gorna, A. S. Turner, Regeneration of bicortical defects in the iliac crest of estrogen-deficient sheep, using new biodegradable polyurethane bone graft substitutes. J Biomed Mater Res Part A 2006; 77 (4): 802.

5. N. M. K. Lamba, K. A. Woodhouse, S. L. Cooper. Polyurethanes in Biomedical Applications. CRC Press LLC. Boca Raton, FL. 1998.

6. P. A. Gunatillake, G. F. Meijs, S. J. McCarthy, R. Adhikari, Poly(dimethylsiloxane)/poly(hexamethylene oxide) mixed macrodiol based polyurethane elastomers. I. Synthesis and properties. J Appl Polym Sci 2000; 76 (14): 2026.

7. V. Jašo, M. Cvetinov, S. Rakić, Z. S. Petrović. Bio-plastics and elastomers from polylactic acid/thermoplastic polyurethane blends. J Appl Polym Sci 2014; 131 (22): DOI:

10.1002/app.41104

8. Z. S. Petrović, Y. Xu, J. Milić, G. Glenn, A. Klamczynski, Biodegradation of Thermoplastic Polyurethanes from Vegetable Oils. J Polym Environment 2010; 18 (2): 94.

9. D. W. Liang, T. Zhang, H. H. Fang, J. He. Phthalates biodegradation in the environment. Appl Microbiol Biotechnol 2008; 80 (2): 183.

\section{FIGURE CAPTIONS}

Figure 1.Mineralization of pure PLA, TPU and their blends.

Figure 2. Mineralization of PLA, TPU and their blends during first 20 days.

Figure 3. Rate of biodegradation of PLA, TPU and their blends. 


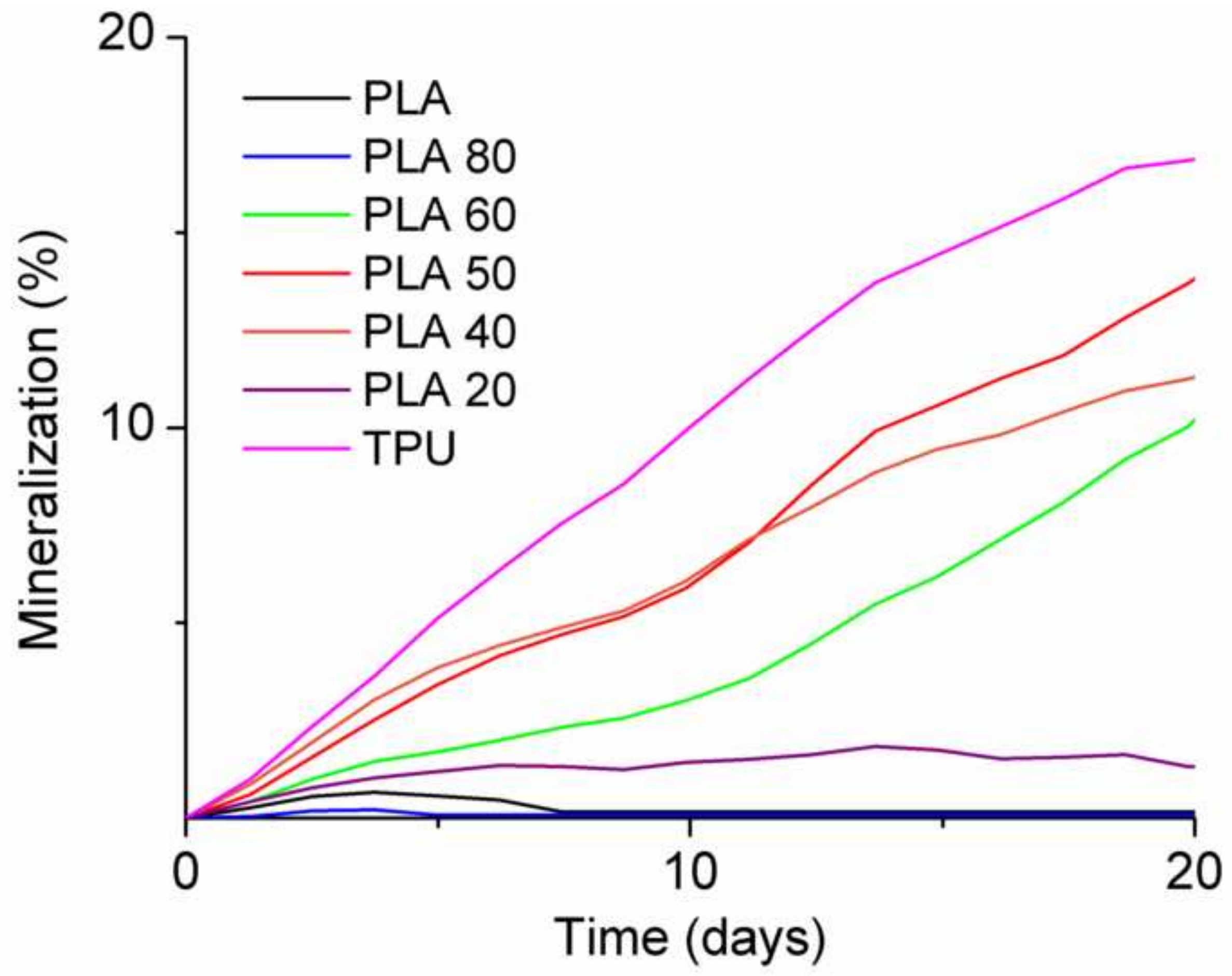




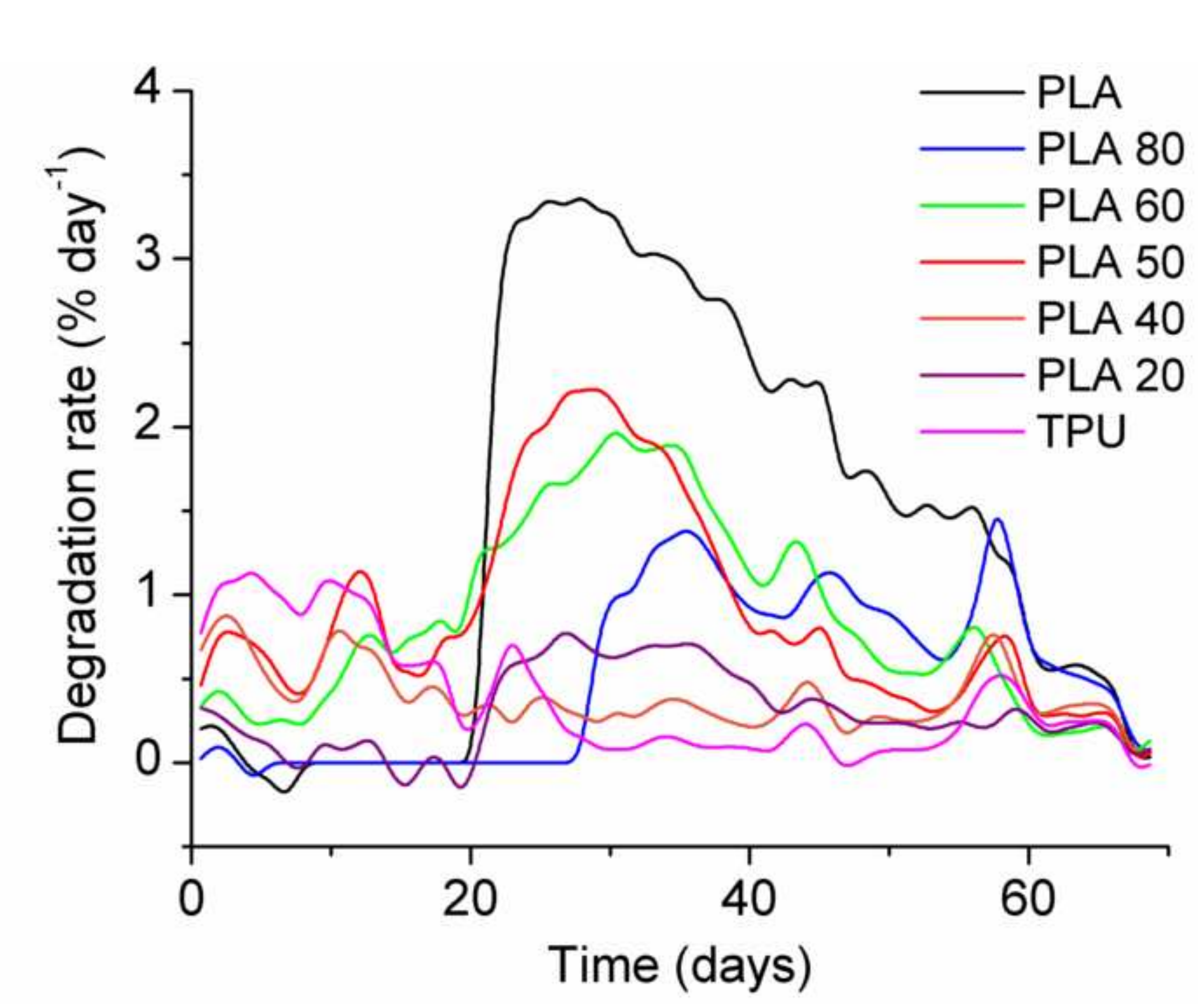

